\title{
The Second National Sun Survey: Workshop report
}

\author{
Bronwen Waller, MHSc (1); Erin Pichora, MSc (1); Loraine Marrett, PhD (1,2)
}

\section{Introduction}

Ultraviolet radiation (UVR) from the sun is the major cause of skin cancer. An estimated 77600 Canadians are diagnosed with skin cancer each year, making it the most common form of cancer. ${ }^{1}$ Developing public health programs to decrease its incidence by reducing unnecessary sun exposure requires accurate, up-to-date information about how much time people spend in the sun, their use of sun protection, and their knowledge, attitudes and behaviours concerning tanning, sun exposure and sun protection.

The Second National Sun Survey (NSS2) was conducted in 2006, 10 years after the Canadian National Survey on Sun Exposures and Protective Behaviours, 1996 (NSS1), ${ }^{2}$ under the auspices of the National Skin Cancer Prevention Committee of the Canadian Strategy for Cancer Control. The NSS2 was designed to estimate:

- current levels of sun exposure, protective behaviours and use of tanning equipment in adults $16+$ by age, sex and region (province or aggregations thereof);

- current levels of sun exposure and protective behaviours in children aged 1-12 for Canada as a whole; and

- levels of knowledge, attitudes and beliefs about sun safety in adults $16+$ by age, sex and region.

The NSS2 was also designed to compare levels of sun exposure and protective behaviours in Canadian adults aged 16 years and older by age and sex between the NSS 2 and the NSS1.
For the first three objectives, a sample of 7121 Canadian adults (the base sample), was interviewed by telephone, with a questionnaire that encompassed all specified areas of interest. To address the fourth objective, an additional 2115 adults (the comparison sample) were asked a reduced set of questions from the NSS1.

An important objective of the survey is knowledge transfer. Therefore, components such as promoting awareness of the survey and its data, enhancing capacity to analyze the data, and producing reports to meet the needs of a wide range of stakeholders, including public health professionals, health promotion experts, planners, policy-makers and sun safety researchers were included in the survey design. To this end, a one-day workshop was held on September 19th 2007 in Toronto. Thirty people attended by invitation, including specialist speakers, project staff and those with policy, program planning, and analysis skills to represent regions across Canada (the participants are listed at the end of this report).

\section{NSS2 workshop planning and objectives}

The planning committee included representatives from the National Skin Cancer Prevention Committee, co-investigators and collaborators on the NSS2 project, and study staff. The objectives of the workshop were to:

- share basic design features and preliminary descriptive results from the NSS2;

- stimulate discussion of priority areas for national and regional analyses;
- identify ways to translate results into products that would be useful for shaping policy and prevention (reduction of UVR exposure and skin cancer prevention);

- explore methods for disseminating these products to enhance impact on health promotion, planning and surveillance; and

- provide a preliminary base sample data file, as well as hands-on dataanalysis training.

To prepare participants, a summary of workshop goals, an overview of the NSS2, and detailed documentation about its content and data files were provided in advance.

\section{Workshop agenda}

The morning session began with an overview of skin cancer and UVR, NSS2 methods and preliminary base sample results by the NSS2 principal investigator, Dr. Loraine Marrett (Cancer Care Ontario). Figures 1 and 2 demonstrate components of survey design and results. Figure 1 illustrates the number of survey respondents by region: one of the NSS2's strengths lies in its relatively large sample sizes for all regions in order to allow extensive and varied analyses at the national level, as well as a broad selection at the regional level. Figure 2 shows the regional variation depicting the proportion of adults who spent at least 2 hours in the sun on a typical summer day.

Dr. Vitali Fioletov (Environment Canada) then shared the results of estimating the UV index for each respondent's location of

Author References

1 Population Studies and Surveillance, Cancer Care Ontario, Toronto, Ontario

2 Department of Public Health Sciences, University of Toronto, Toronto, Ontario

Correspondence: Loraine Marrett, Population Studies and Surveillance, Cancer Care Ontario, University of Toronto, 620 University Ave, Toronto ON M5G 2L7,

Tel.: 416-217-1381, Fax: 416-971-6888, Email: loraine.marrett@cancercare.on.ca

For information on how to access the Second National Sun Survey (NSS2) Public Use Data File, and/or related materials, please email surveillanceunit@cancercare.on.ca 
FIGURE 1

Second National Sun Survey (NSS2) sample sizes by province/region, 2006

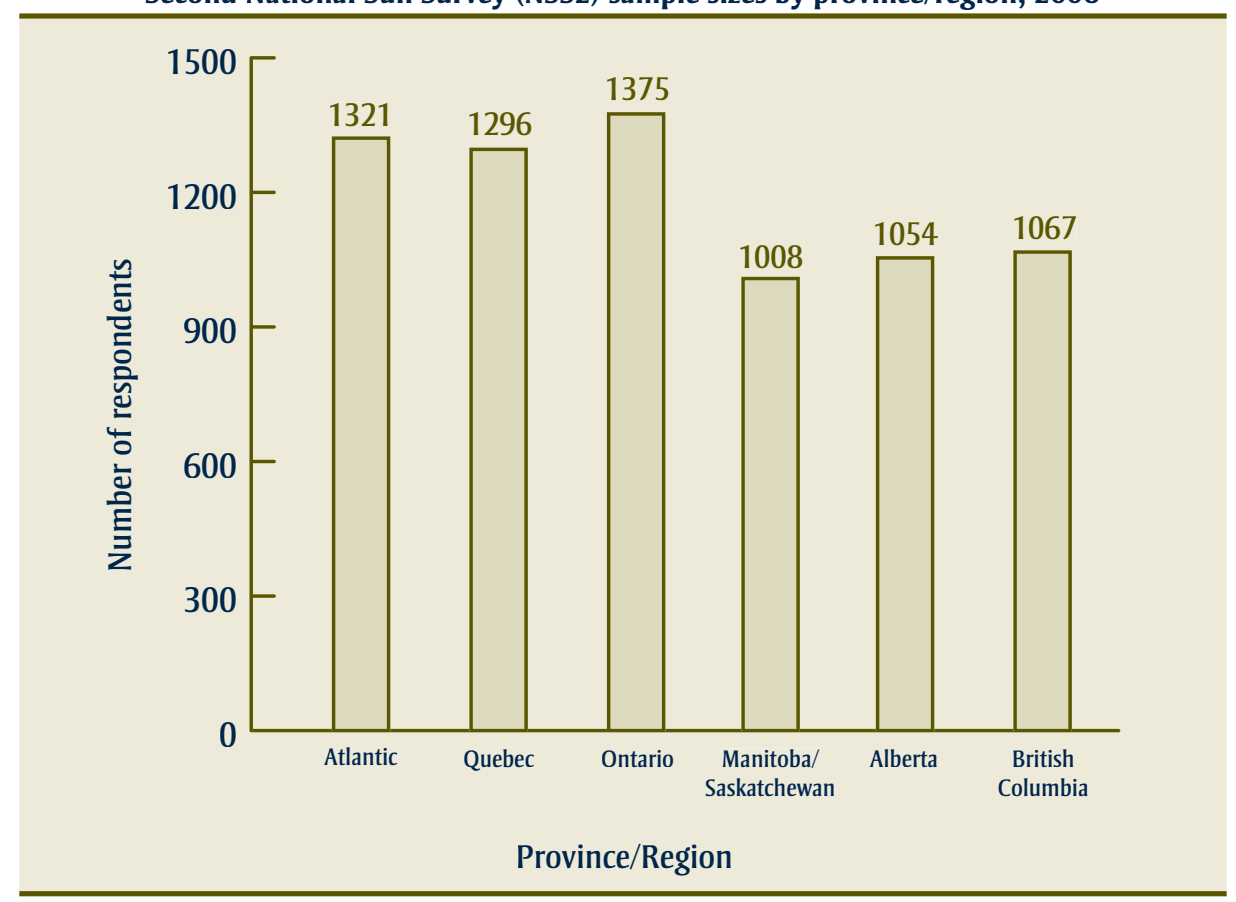

Atlantic Provinces $=$ New Brunswick, Newfoundland/Labrador, Nova Scotia and Prince Edward Island

Source: Second National Sun Survey, 2006

FIGURE 2a

Percent of Canadian adults 16+ who spent at least 2 hours in the sun on a typical summer day by province/region, $2006^{\mathrm{b}}$

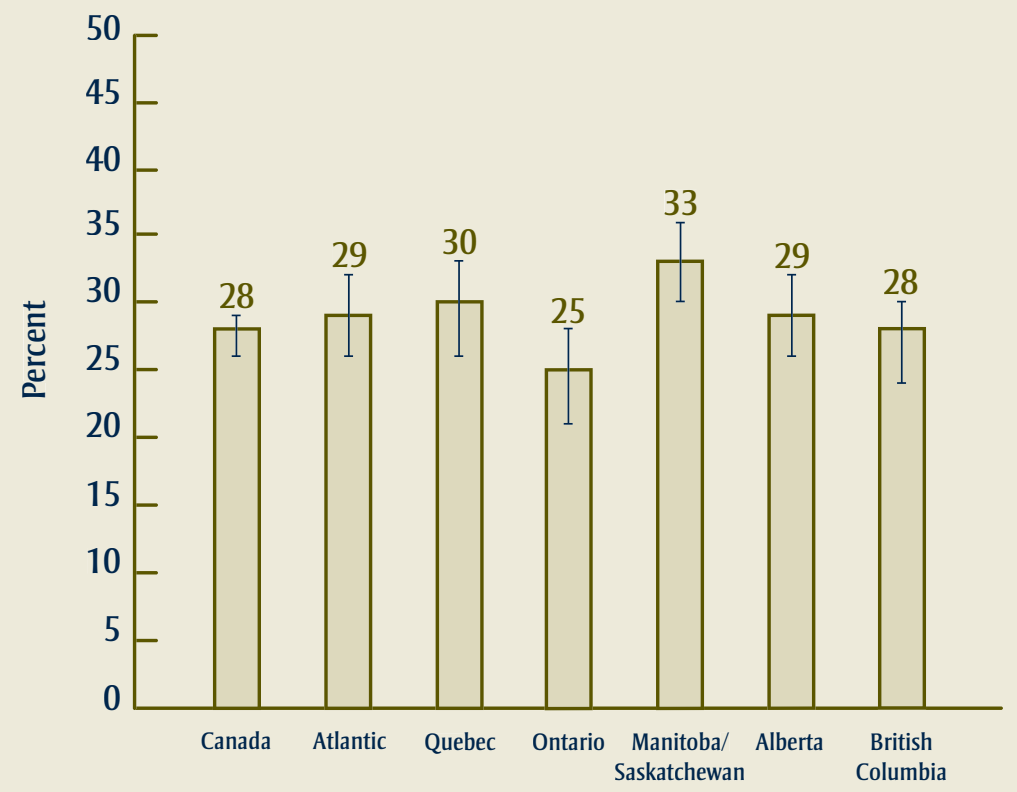

\section{Province/Region}

\footnotetext{
This figure is based on a composite measure (weighted average) of two questionnaire items:

a. Amount of time spent in the sun between 11 a.m. and 4 p.m. on a typical weekday during June, July and August, an

b. Amount of time spent in the sun between 11 a.m. and 4 p.m. on a typical weekend day during June, July and August Age-standardized to the 2001 Canadian population $I=95 \%$ confidence intervals

b Atlantic Provinces $=$ New Brunswick, Newfoundland/Labrador, Nova Scotia and Prince Edward Island Source: Second National Sun Survey, 2006
}

residence during the summer of 2006. These estimates will allow a more comprehensive assessment of individual-level UVR dose, as well as exploration of reported behaviours in relation to climatology. Dr. Scott Leatherdale (Cancer Care Ontario) presented an overview of NSS2 results regarding knowledge, attitudes and beliefs about sun safety.

After lunch, participants split into two groups:

Group 1. NSS2 staff led the 7 data analysts in a hands-on training session covering the following areas:

- basic survey sampling methodology;

- contents of the draft NSS2 Data User Guide, with emphasis on the NSS2 sampling design and computation of weights, calculation of precision indicators (i.e. sampling variance) and data release guidelines;

- structure and contents of the preliminary base sample public use data file; and

- methodology and syntax for generating NSS2 estimates using SAS and SPSS

Analysts were given the preliminary base sample public use data file on $\mathrm{CD}$. The final base sample public use data file was distributed at a second analyst workshop (held in March 2008) that resulted in part from participants' very positive evaluations of the September 2007 workshop and the expression of a need for further analysis training

Group 2. The remaining attendees participated in an interactive discussion session designed to provide study staff with some direction around:

- priority UVR protection issues and how the NSS2 could help address them;

- key target audiences for information/ reports from the NSS2;

- useful and effective characteristics of reports; and 
- methods of ongoing collaboration and communication.

A synopsis of these discussions and main themes is presented in Table 1 .

During this session, Dr. Steve Manske (University of Waterloo) provided an overview of knowledge transfer and exchange. He emphasized the importance of relevant, simply-stated information, as well as interaction taking into account all participants' needs, as the main elements for translation of knowledge into action. ${ }^{3}$

\section{Recommendations}

The NSS2 Workshop participants provided key advice and recommendations about translation of data into useful products to

TABLE 1

Sun safety issues and recommendations from the Second National Sun Survey Workshop

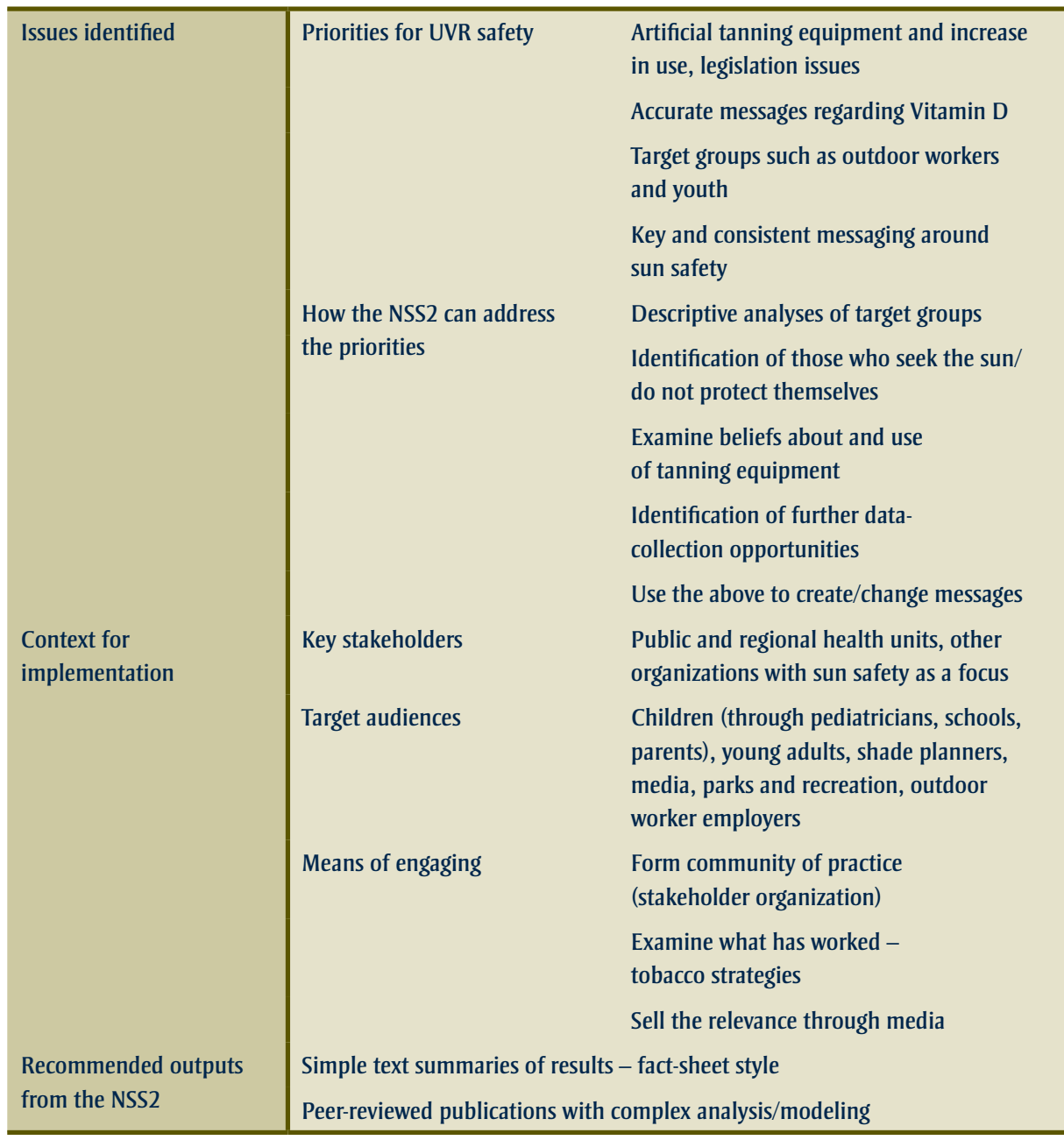

together is tobacco taxation and the denormalization of smoking through social marketing.

Participants suggested that reports with short descriptive chapters and simple text summaries of results would be most effective. Peer-reviewed publications could be used for complex analyses involving modeling, while SunSurv, a data system designed to provide users with access to NSS2 background documentation and basic descriptive results, will be crucial for generating basic descriptive data by nonanalysts. Participants agreed that the NSS2 data could play a key role in motivating sun behaviour change through translating data into useable material for policy-makers, ultimately to aid in the prevention of skin cancer.

\section{Acknowledgements}

This NSS2 workshop was funded by the Canadian Cancer Society (National and Ontario Divisions) as well as the Public Health Agency of Canada. The principal investigator for the NSS2 is Dr. Loraine Marrett, and co-investigators are Dr. Cheryl Rosen, Dr. Joel Claveau, Dr. Marc Rhainds, David Northrup, and Jennifer Fergenbaum.

The authors would also like to acknowledge the assistance of the National Skin Cancer Prevention Committee, whose members acted in a steering capacity, and the Institute for Social Research (York University), as well as Jolie-Coeur and Associates (Quebec) for administering the survey.

Michael Spinks (South East Local Health Integration Network; Cancer Care Ontario) provided invaluable guidance for analysis planning, and structuring and facilitating the workshop analysis session. Yen Borrego, Sandrene Chin Cheong and Greg Kennedy of Cancer Care Ontario are also acknowledged for their support around workshop planning, data management and analysis, and preparation of graphical materials. 


\section{Workshop participants}

Riaz Alvi (Saskatchewan Cancer Agency), Rosemary Boyle (Canadian Cancer Society, New Brunswick Division), Doreen Callander (Canadian Cancer Society, Saskatchewan Division), Heather Chappell (Canadian Cancer Society), Christina Chociolko (National Collaborating Centre for Environmental Health), Vitali Fioletov (Environment Canada), Lynn From (Women's College Hospital), Irene Gallagher (Canadian Cancer Society, Ontario Division), Suzanne Gingras (Institut national de santé publique du Québec), Jane Griffith (Cancer Care Manitoba), * Scott Leatherdale (Cancer Care Ontario), Tim Lee (BC Cancer Research Centre), Sylvia Leonard (Canadian Cancer Society, Ontario Division), *Steve Manske (University of Waterloo), *Loraine Marrett (Cancer Care Ontario), *David Northrup (Institute for Social Research), Corinne Parker (Alberta Cancer Board), *Erin Pichora (Cancer Care Ontario), *Judy Purcell (Cancer Care Nova Scotia), Steven Quantz (Alberta Cancer Board), Pascale Reinhardt (Health Canada), *Marc Rhainds (Institut national de santé publique du Québec), * Cheryl Rosen (Toronto Western Hospital), Holly Smith (Canadian Cancer Society, PEI Division), *Michael Spinks (South East Local Health Integration Network and Cancer Care Ontario), Sharon Storoschuk (Canadian Cancer Society, BC and Yukon Division), *Bronwen Waller (Cancer Care Ontario), Gordon Walsh (Cancer Care Nova Scotia), Lin Xue (Cancer Care Manitoba).

${ }^{*}$ Member of workshop planning committee/ NSS2 staff/co-investigators

\section{References}

1. Canadian Cancer Society/National Cancer Institute of Canada. Canadian Cancer Statistics. Toronto: Canadian Cancer Society/ National Cancer Institute of Canada, 2008.

2. Lovato C, Shoveller J, Rivers J (eds). Final Report: National Survey on Sun Exposure and Protective Behaviours. Institute of Health Promotion Research: University of British Columbia, 1997.

3. Graham ID, Logan J, Harrison MB, et al. Lost in knowledge translation: time for a map? J Contin Educ Health Prof. 2006;26(1):13-24. 